\title{
Histologically confirmed distant metastatic urothelial carcinoma from the urinary bladder: a retrospective review of one institution's 20-year experience
}

\author{
Youngeun Yoo, Junghye Lee, Heae Surng Park, Min-Sun Cho, Sun Hee Sung, Sanghui Park, Euno Choi \\ Department of Pathology, Ewha Womans University College of Medicine, Seoul, Korea
}

\begin{abstract}
Background: Urothelial carcinoma (UC) accounts for roughly $90 \%$ of bladder cancer, and has a high propensity for diverse differentiation. Recently, certain histologic variants of UC have been recognized to be associated with unfavorable clinical outcomes. Several UC studies have also suggested that tumor budding is a poor prognostic marker. Distant metastasis of UC after radical cystectomy is not uncommon. However, these metastatic lesions are not routinely confirmed with histology. Methods: We investigated the histopathologic features of 13 cases of UC with biopsy-proven distant metastases, with a special emphasis on histologic variants and tumor budding. Results: Lymph nodes $(6 / 13,46 \%)$ were the most common metastatic sites, followed by the lung $(4 / 13,31 \%)$, liver $(4 / 13,31 \%)$, and the adrenal gland $(2 / 13,15 \%)$. The histologic variants including squamous $(n=1)$, micropapillary $(n=4)$, and plasmacytoid $(n=1)$ variants in five cases of UC. Most histologic variants (4/5, 80\%) of primary UCs appeared in the metastatic lesions. In contrast, high-grade tumor budding was detected in six cases (46\%), including one case of non-muscle invasive UC. Our study demonstrates that histologic variants are not uncommonly detected in distant metastatic UCs. Most histologic variants seen in primary UCs persist in the distant metastatic lesions. In addition, high-grade tumor budding, which occurs frequently in primary tumors, may contribute to the development of distant metastasis. Conclusions: Therefore, assessing the presence or absence of histologic variants and tumor budding in UCs of the urinary bladder, even in non-muscle invasive UCs, may be useful to predict distant metastasis.
\end{abstract}

Key Words: Bladder neoplasms; Distant metastasis; Urothelial carcinoma; Histologic variant; Tumor budding

Received: September 25, 2020 Revised: October 9, 2020 Accepted: October 19, 2020

Corresponding Author: Euno Choi, MD, Department of Pathology, Ewha Womans University College of Medicine, 260 Gonghang-daero, Gangseo-gu, Seoul 07804, Korea Tel: +82-2-2650-5194, Fax: +82-2-2650-2879, E-mail: euno1103@hanmail.net

Bladder cancer is the sixth most prevalent cancer and ninth leading cause of cancer death in men worldwide [1]. Urothelial carcinoma comprises approximately $90 \%$ of bladder cancers, and exhibits a great propensity for divergent differentiation [2]. Reflecting this morphologic diversity, histologic variants have been proposed to define urothelial carcinoma with distinctive histomorphology that differs from that of conventional urothelial carcinoma [2,3]. The World Health Organization (WHO) recently classified urothelial cancers into 13 histologic variants [4]. There is also accumulating evidence regarding the clinical relevance of histologic variants with regard to prognosis and response to treatment. Therefore, it is becoming increasingly important to recognize the histologic variant [3,5]. Several histologic variants, including squamous differentiation, micropapillary carcinoma, plasmacytoid, sarcomatoid, and nested variants, are as- sociated with unfavorable clinical outcomes [6-11]. These histological variants frequently persist, even in metastatic tumors [6]. In addition to histologic variants, tumor budding has also been proposed as a poor prognostic indicator [12,13]. Tumor budding is defined by the presence of single tumor cells or clusters of fewer than five cancer cells at the invasive front. It has been recognized as an adverse prognostic factor in several types of cancer, especially in colorectal cancer [14-17]. Tumor budding is considered to represent the epithelial to mesenchymal transition by which tumor cells achieve migratory and invasive properties for metastasis [18].

Radical cystectomy is the gold standard treatment for patients with muscle-invasive bladder cancer. Based on recent data, patients with non-muscle invasive bladder cancer harboring micropapillary carcinoma or plasmacytoid variants are also considered 
candidates for early radical cystectomy due to the aggressive clinical course $[2,6,9]$.

Distant metastasis occurs in 10\%-29\% of patients with bladder cancer during their disease course [19]. Once distant metastasis develops, the long-term prognosis is generally poor [20]. Certain histologic variants in bladder cancer, including micropapillary carcinoma, the plasmacytoid variant, and the sarcomatoid variant, are associated with a high tendency to develop distant metastasis $[8,10,21,22]$. However, few studies have evaluated the histopathologic parameters and prevalence of histologic variants in metastatic urothelial carcinoma of distant metastatic sites, because histologic confirmation is rarely performed in this setting.

In this study, we analyzed the histopathologic findings of 13 cases of urothelial carcinomas of the urinary bladder with histologically confirmed distant metastases. We studied the correlation between the histomorphology of the primary tumors and that of subsequent distant metastases with an emphasis on the histologic variants. We also assessed various histopathologic parameters, including tumor budding.

\section{MATERIALS AND METHODS}

\section{Case selection}

A retrospective chart review was conducted of 477 patients with urothelial carcinoma of the urinary bladder who were treated by radical cystectomy at Ewha Womans University Mokdong Hospital between 1997 and 2017. Twelve of the 477 patients with histologically confirmed distant metastasis were selected for inclusion in this study. Additionally, one patient who was diagnosed with urothelial carcinoma by transurethral resection of the bladder and needle biopsy of the liver was included. The following clinical data were obtained from the medical records: sex, age, procedure, primary tumor size, metastatic site, biopsy site, tumor stage, and follow-up data.

\section{Histologic evaluation}

All hematoxylin and eosin stained slides from the 13 patients were reviewed by two uropathologists (SP and EC). The stage, grade, and histologic variants were reclassified according to the Cancer Staging Manual of the American Joint Committee on Cancer, 8th edition and the 2016 WHO/International Society of Urologic Pathology grading system [4,23]. Histologic variants were compared between the matched primary and metastatic tumors. Data concerning lymphovascular invasion, perineural invasion, accompanying urothelial carcinoma in situ, and tumor budding were recorded. Tumor budding was assessed with hema- toxylin and eosin staining. Tumor budding was defined by the presence of isolated tumor cells or small groups of fewer than five cancer cells at the invasion front [14]. Tumor budding foci were counted in hot spots under $\times 200$ magnification. Tumor budding was regarded as high-grade when 10 or more budding foci were identified [24].

\section{IHC staining}

Immunohistochemical (IHC) staining for $\alpha$-fetoprotein (AFP; 1:1,000, clone C3, mouse monoclonal, Novocastra, Newcastle upon Tyne, UK) was performed on 4-um thick slides using an automated immunostainer (BOND MAX, Leica Biosystem, Newcastle upon Tyne, UK) following the manufacturer's protocol. We performed immunostaining for AFP in one case with hepatic feature. In this case, most tumor cells demonstrated cytoplasmic expression for AFP.

\section{RESULTS}

\section{Baseline features}

The clinical features of the 13 patients are summarized in Table 1 . All 13 cases arose primarily from the urinary bladder. Ten patients $(77 \%)$ were men, and the median age was 59 years (range, 38 to 76 years). Twelve patients were treated with radical cystectomy for urothelial carcinoma of the urinary bladder, and one patient underwent a transurethral resection of bladder tumor with concurrent liver metastasis. The primary tumor size (maximum tumor diameter by pathological report) ranged from 0.5 to $8.5 \mathrm{~cm}$ (median, $3.5 \mathrm{~cm}$ ). Clinically, eight (62\%) distant metastatic lesions presented as solitary masses, while multiple metastatic lesions were detected in five cases (38\%). Lymph nodes were the most frequent distant metastatic site (46\%), followed by the lung (38\%), liver (31\%), and adrenal gland (15\%). The biopsy sites of distant metastatic tumors are presented in Table 1. Regarding pathologic tumor stage (pT), 10 cases (77\%) were classified as muscle-invasive urothelial carcinoma (pT2T3), and three (23\%) were classified as non-muscle-invasive urothelial carcinoma (pT1). The median follow-up duration was 47 months (range, 8 to 151 months). The median interval from the initial diagnosis of the primary lesion to that of distant metastasis was 25 months (range, 0 to 123 months).

\section{Histopathologic features}

The overall histopathologic findings are summarized in Table 2. Eight cases (62\%) presented with conventional high-grade urothelial carcinoma. Histologic variants were present in the pure 
Table 1. Baseline patient characteristics

\begin{tabular}{|c|c|c|c|c|c|c|c|c|c|}
\hline Case & Sex & Age (yr) & Operation & $\begin{array}{l}\text { Primary tumor } \\
\text { size }(\mathrm{cm})\end{array}$ & $\begin{array}{l}\text { Clinical distant } \\
\text { metastatic sites }\end{array}$ & $\begin{array}{l}\text { Bx site of distant } \\
\text { metastatic lesion }\end{array}$ & pTNM & $\mathrm{F} / \mathrm{U}(\mathrm{mo})$ & $\begin{array}{c}\text { Interval } \\
\text { (mo) }\end{array}$ \\
\hline 1 & M & 59 & $\mathrm{RC}$ & 8.5, multiple & Lung & Lung & pT2N0M1b & 47 & 19 \\
\hline 2 & M & 38 & $\mathrm{RC}$ & $0.5(\times 2)$ & Liver, neck LN & Neck LN & pT2N0M1b & 46 & 25 \\
\hline 3 & M & 76 & TURBT & NA & Liver & Liver & NA & 22 & 0 \\
\hline 4 & M & 55 & $\mathrm{RC}$ & 3.5, multiple & Colon, peritoneal seeding & Colon & pT3N2M1b & 8 & 8 \\
\hline 5 & M & 58 & $\mathrm{RC}$ & 2.3, multiple & $\begin{array}{l}\text { Liver, adrenal gland, inguinal, retrocaval, } \\
\text { aortocaval LNs }\end{array}$ & Inguinal LN & pT3N2M1b & 30 & 13 \\
\hline 6 & M & 74 & $\mathrm{RC}$ & 1.5 & Axillary LN & Axillary LN & pT2N0M1a & 151 & 123 \\
\hline 7 & $\mathrm{~F}$ & 59 & $\mathrm{RC}$ & 3.5 & Liver & Liver & pT2N1M1b & 43 & 28 \\
\hline 8 & M & 64 & $\mathrm{RC}$ & 4.5 & Adrenal gland & Adrenal gland & pT3N0M1b & 68 & 6 \\
\hline 9 & $\mathrm{~F}$ & 64 & $\mathrm{RC}$ & 6 , multiple & $\begin{array}{l}\text { Lung, vagina, right rectus muscle, } \\
\text { mediastinal LN }\end{array}$ & Lung & pT1N0M1b & 76 & 60 \\
\hline 10 & M & 62 & $\mathrm{RC}$ & 2.5 & Neck LN & Neck LN & pT1NXM1a & 64 & 31 \\
\hline 11 & $\mathrm{~F}$ & 69 & $\mathrm{RC}$ & 2.5 & Lung & Lung & pT3N0M1b & 90 & 61 \\
\hline 12 & M & 55 & $\mathrm{RC}$ & 3.8 & Lung (both) & Lung & pT3N1M1b & 16 & 16 \\
\hline 13 & M & 59 & $\mathrm{RC}$ & 4 & Inguinal LN & Inguinal LN & pT1N0M1a & 61 & 58 \\
\hline
\end{tabular}

Bx, biopsy; F/U, follow-up; RC, radical cystectomy; LN, lymph node; TURBT, transurethral resection of bladder tumor; NA, non-applicable.

Table 2. Comparison of histologic components between primary and distant metastatic tumors

\begin{tabular}{|c|c|c|c|c|c|c|c|}
\hline $\begin{array}{l}\text { Case } \\
\text { No. }\end{array}$ & Stage & Primary tumor histology & LVI & $\mathrm{Nl}$ & $\mathrm{ClS}$ & TB & Distant metastatic tumor histology \\
\hline 1 & pT2N0M1b & Conventional UC with MPC (3\%) & + & - & - & + & MPC (100\%) \\
\hline 2 & pT2N0M1b & Conventional UC & - & + & + & - & Conventional UC \\
\hline 3 & NA & AFP-producing type UC & + & NA & - & NA & AFP-producing type UC \\
\hline 4 & pT3N2M1b & Conventional UC with MPC (10\%) and squamous differentiation (70\%) & + & + & + & + & Squamous differentiation (100\%) \\
\hline 5 & pT3N2M1b & Conventional UC & + & + & + & - & Conventional UC \\
\hline 6 & pT2N0M1a & Plasmacytoid variant (100\%) & - & - & + & - & Plasmacytoid variant (100\%) \\
\hline 7 & pT2N1M1b & Conventional UC with MPC (20\%) & + & - & + & + & Conventional UC \\
\hline 8 & pT3N0M1b & Conventional UC & + & + & + & + & Conventional UC \\
\hline 9 & pT1N0M1b & Conventional UC & - & - & + & + & Conventional UC \\
\hline 10 & pT1NXM1a & Conventional UC & + & - & + & - & Conventional UC with squamous (1\%) \\
\hline 11 & pT3N0M1b & Conventional UC with MPC (3\%) & + & + & + & + & Conventional UC with mpc (1\%) \\
\hline 12 & pT3N1M1b & Conventional UC & + & + & + & - & Conventional UC \\
\hline 13 & pT1N0M1a & Conventional UC & - & - & - & - & Conventional UC \\
\hline
\end{tabular}

LVI, lymphovascular invasion; NI, neural invasion; CIS, carcinoma in situ; TB, tumor budding; UC, urothelial carcinoma; MPC, micropapillary carcinoma component; NA, non-applicable; AFP, $\alpha$-fetoprotein.

form in one case $(1 / 13,8 \%)$ and mixed with conventional highgrade urothelial carcinoma in four cases $(4 / 13,31 \%)$. Four of these mixed cases (cases 1, 4, 7, and 11) had a micropapillary carcinoma component (3\%-20\%) and 1 (case 4) had concurrent squamous differentiation (70\%). One case was diagnosed as an AFP-producing urothelial carcinoma. Lymphovascular and perineural invasion were identified in nine cases (69\%) and six cases (46\%), respectively. Most cases (77\%) had concomitant urothelial carcinoma in situ. High-grade tumor budding was identified in six cases (46\%) (cases 1, 4, 7, 8, 9, and 11) (Fig. 1).

Histologic evaluation of the metastatic carcinomas was available in all 13 cases. Histologic variants were noted in the metastatic sites in five of 13 cases (38\%). Most of these histologic vari- ants $(80 \%, 4 / 5)$ were also identified in the primary tumors. In case 1 , the metastatic tumor was entirely composed of micropapillary carcinoma, which was focally present (3\%) in the corresponding primary tumor (Fig. 2A, B). Although the primary tumor of case 4 had components of micropapillary carcinoma $(10 \%)$ and squamous differentiation (70\%), the corresponding metastatic tumor only demonstrated carcinoma cells with squamous differentiation (Fig. 2C, D). The primary tumor in case 6 was considered a plasmacytoid variant, which was maintained at the metastatic site (Fig. 2E, F). In case 10, the primary tumor was a conventional high-grade urothelial carcinoma, which persisted in the corresponding metastatic tumor along with focal squamous differentiation (1\%). Squamous differentiation was 

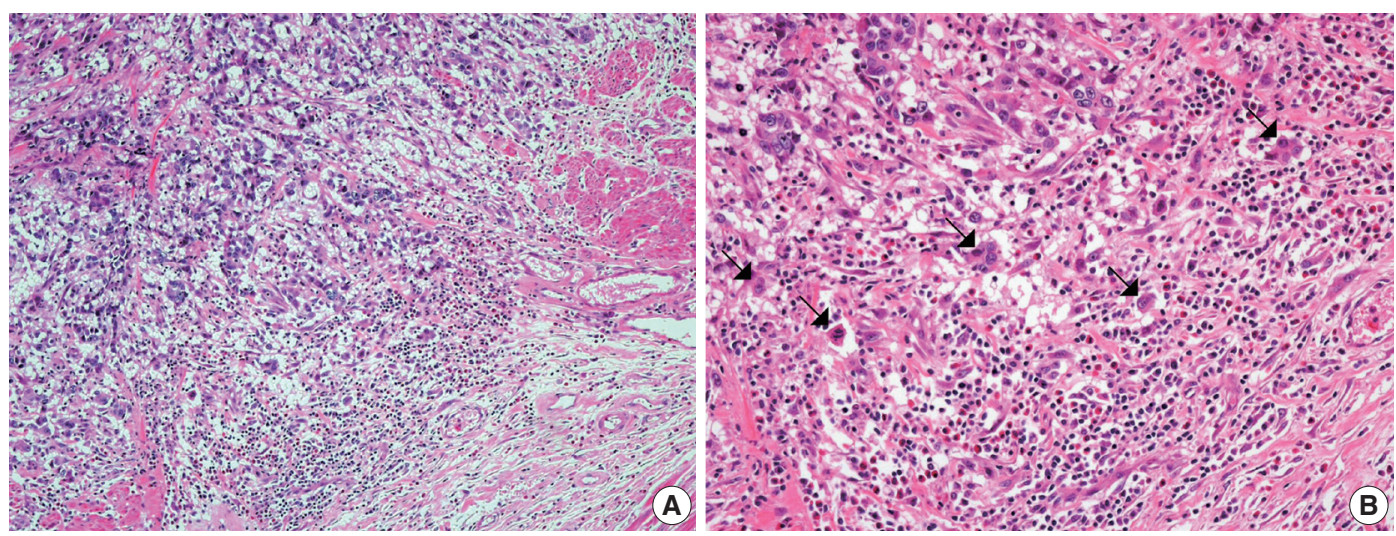

Fig. 1. Representative morphology of tumor budding at the invasive front. (A, B) Hematoxylin and eosin staining shows single or small clusters of tumor cells with up to 5 cells per cluster (arrows) detached from the main tumor mass.

not identified in the corresponding primary tumor. In case 11, the primary tumor showed predominant conventional highgrade urothelial carcinoma with a small micropapillary carcinoma component (3\%). The corresponding metastatic tumor also revealed small amounts of micropapillary carcinoma component (1\%). In case 3, both primary and metastatic tumors were AFPproducing urothelial carcinomas (Fig. 3). There were no remarkable differences in the histomorphologic findings between primary and corresponding metastatic tumors in seven cases (cases $2,5,7,8,9,12$, and 13) that were primarily diagnosed as conventional high-grade urothelial carcinomas.

\section{DISCUSSION}

Regional lymph node metastasis occurs in approximately $30 \%$ of bladder cancers with muscle invasion (pT2) and $60 \%$ of bladder cancers with extravesical fat extension (pT3-pT4) [25]. The survival outcome of muscle-invasive bladder cancer (pT2pT4) with regional lymph node metastasis is poor with an $11 \%$ 5-year survival rate [26]. Distant metastasis of bladder cancer develops in 10\%-29\% of patients [19]. Once distant metastasis occurs, survival outcome is very poor with a median survival time of 8.5 months [27].

A previous autopsy study of 367 patients with muscle-invasive bladder cancer demonstrated that the frequency of metastases was strongly associated with local tumor extension and regional lymph node metastases. There was no difference in the frequency of metastases between 308 transitional cell carcinomas and 38 squamous cell carcinomas [28] .

In addition, a recent study on the metastatic patterns of bladder cancer showed that advanced $\mathrm{T}$ category or atypical histologic features were linked to an early occurrence of distant metastasis
[29]. In this study, we confirmed that histologic variants were not uncommonly detected in urothelial carcinomas with distant metastasis. In addition, these histologic variants were usually present in both primary and accompanying metastatic tumors. Five of the 13 cases included histologic variants, one in pure form and four in mixed forms. The histologic variants of primary tumors were preserved in four corresponding metastatic tumors ( $4 / 5$, $80 \%$ ). The following histologic variants of the primary tumors were maintained in the metastatic tumors: micropapillary carcinoma variant $(2 / 4,50 \%)$, squamous differentiation $(1 / 1,100 \%)$, and plasmacytoid variant $(1 / 1,100 \%)$.

Additionally, we present a case of AFP-producing urothelial carcinoma, which has only been described before in several case reports [30,31]. In the previously reported cases, most patients had an increased level of serum AFP, which is closely correlated with tumor burden and may serve as a tumor marker. All tumors also showed aberrant AFP expression by IHC [30,31]. El-Bahrawy et al. [31] emphasized that although AFP-producing urothelial tumors are extremely rare, it is important to be aware of this tumor type because it requires a different chemotherapeutic regimen and has a different prognosis than do AFP-producing germ cell tumors.

Previous studies reported that histologic variants are associated with an advanced disease stage [32]. Recognition of histologic variants is important because they commonly persist in metastatic tumors and represent the association between primary and corresponding metastatic tumors $[6,33]$. However, to the best of our knowledge, no prior studies have evaluated the frequency of histologic variants in metastatic urothelial carcinomas compared to that of primary urothelial carcinomas of the urinary bladder. In this study, we confirmed that the histologic variants of urothelial carcinoma, even if focally present, frequently metas- 

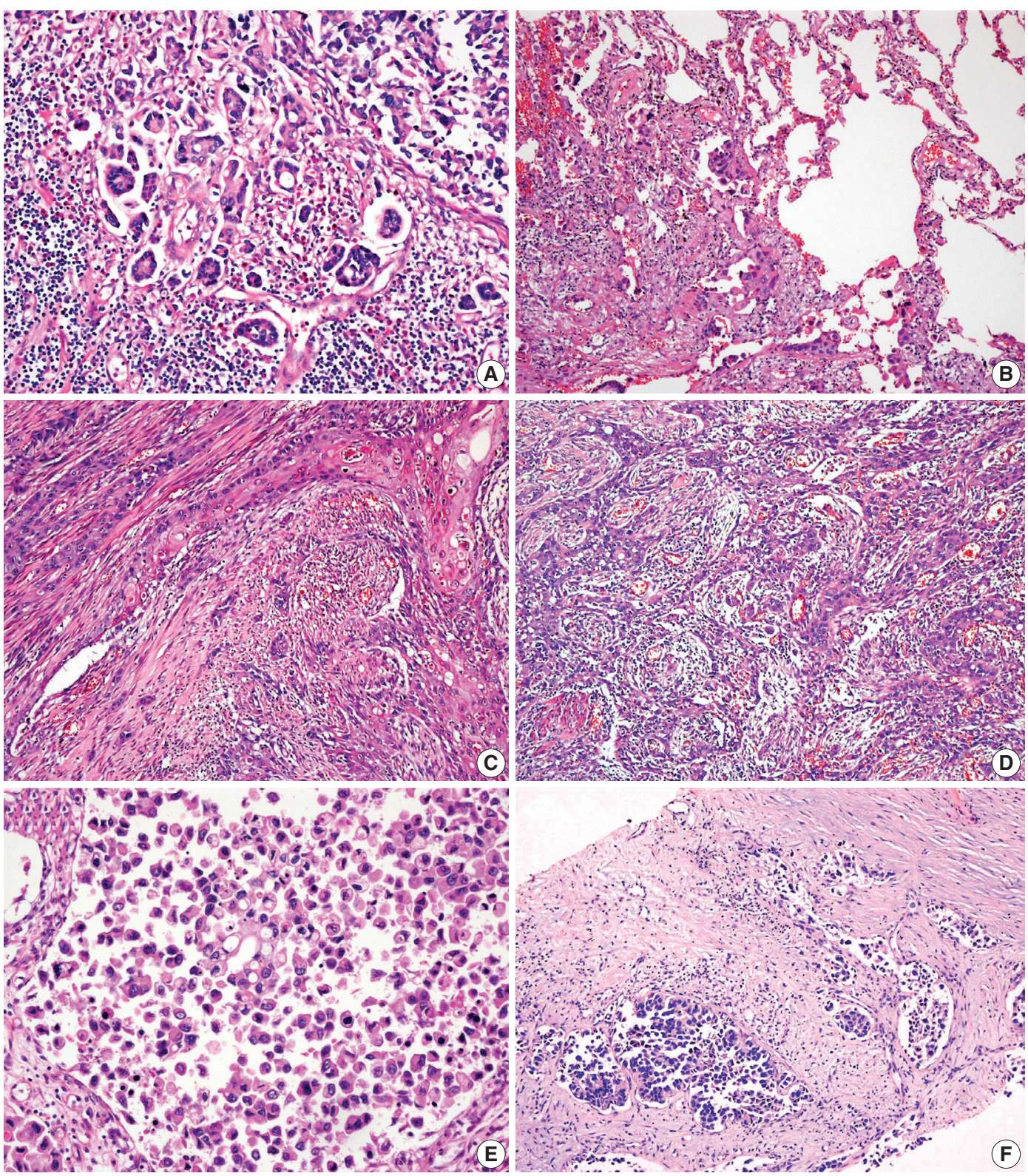

Fig. 2. Histologic features of three histologic variants that are concurrently displayed in both primary and metastatic lesions. (A) In case 1, the primary bladder tumor focally shows a micropapillary carcinoma component characterized by small tight nests or balls with reverse polarity within lacunae. (B) A subsequent pulmonary metastatic lesion is entirely comprised of a micropapillary carcinoma component. (C) The primary bladder tumor in case 4 shows marked squamous differentiation. (D) Squamous differentiation seen in the primary tumor of case 4 is preserved in the subsequent colonic metastatic lesion. (E) In the primary bladder tumor of case 6, the tumor cells are entirely composed of highly atypical discohesive plasmacytoid cells arranged in a solid sheet-like arhitecture. These histologic findings are compatible with plasmacytoid urothelial carcinoma. (F) Plasmacytoid morphology was maintained in the metastatic tumor cells in the axillary lymph node.

tasize to distant organs. Therefore, histologic examination of urothelial carcinomas in the urinary bladder should be performed with an emphasis on the presence or absence of histologic variants.

In this study, high-grade tumor budding was detected in almost half of all cases, including five muscle-invasive and one non-muscle-invasive urothelial carcinomas. Lymphovascular invasion was present in all five muscle-invasive urothelial carcinoma cases with high-grade tumor budding. Tumor budding is a histologic phenomenon encountered at the invasive edge of various carcinomas. It represents the epithelial-mesenchymal transition by which tumor cells obtain the ability to disseminate and metastasize $[15,18]$. Tumor budding is associated with aggressive bi- 

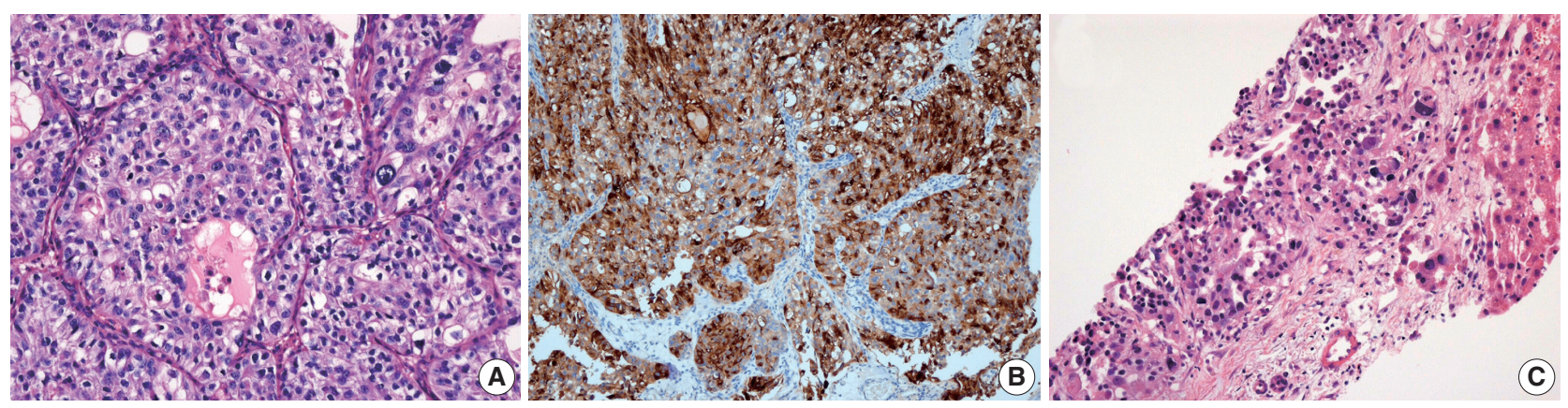

Fig. 3. Histologic and immunohistochemical findings of case 3. (A) In the primary tumor, polygonal tumor cells are arranged in large nests and have highly pleomorphic nuclei and abundant clear to eosinophilic cytoplasm. (B) On immunohistochemistry, most tumor cells show strong positivity for $\alpha$-fetoprotein (AFP), implicating tumor-derived AFP production. (C) Representative histologic features of hepatic metastatic lesion.

ologic characteristics in various types of carcinomas, including frequent lymphovascular invasion, advanced stage, and distant metastasis $[15,18]$. In colorectal cancer, high tumor budding has been identified as a predictive biomarker for distant metastasis at the locally advanced stage $[18,34]$. In this study, six cases (5 which were muscle-invasive bladder cancer) showed lymphovascular invasion that was associated with high-grade tumor budding. A few studies have investigated the clinicopathologic significance of tumor budding in bladder cancer $[12,13]$. In two previous reports, high tumor budding was suggested to be an adverse pathologic indicator to predict stage progression in both non-muscle-invasive and muscle-invasive bladder cancers [12,13]. Further, larger studies are needed to clarify the association between tumor budding and distant metastasis of bladder cancer.

Distant metastasis of non-muscle-invasive urothelial carcinoma is extremely rare. In a review of over 1,000 patients with bladder cancer (irrespective of stage), Metthews et al. [35] described distant metastases in only nine superficial urothelial carcinomas, including three diagnosed as stage pTa. In the current study, we describe distant metastasis in three cases of non-muscle-invasive urothelial carcinoma, which were of the pT1 stage at the time of radical cystectomy. None of these cases showed lymph node metastasis or local recurrence during follow-up. All three of these cases were conventional urothelial carcinomas, and two cases exhibited concomitant carcinoma in situ. Two of the three cases presented with multifocal stromal invasion. Lymphovascular invasion was detected in one case, and high-grade tumor budding was identified in another case. These findings suggest that carcinoma in situ, multifocal stromal invasion, lymphovascular invasion, or high-grade tumor budding may be important histopathologic factors that affect distant metastasis, even in early stage bladder cancer.

There are several limitations in this study. First, the number of cases was small. Only 13 cases of metastatic urothelial carcinoma were included. The small sample size made it difficult to conduct appropriate statistical tests, which allow for adequate statistical power. In addition, we could not assess the effect of the presence or absence of histologic variants and tumor budding on distant metastasis because the current study only included patients who developed distant metastasis. Second, our study yielded limited results on the impact of tumor budding on distant metastasis in urothelial carcinoma, because this study consisted of a small number of metastatic urothelial carcinoma cases and did not investigate tumor budding in patients without distant metastasis. In contrast, few studies have evaluated the prognostic value of tumor budding in urothelial carcinomas $[12,13]$. In addition, there are no well-defined criteria to evaluate tumor buddings in urothelial carcinomas. Therefore, we assessed tumor budding according to the International Tumor Budding Consensus Conference criteria [24], which is a standardized scoring system for tumor budding in colorectal cancers. To the best of our knowledge, this is the first study to investigate the presence of tumor budding in metastatic urothelial carcinomas. Further studies with larger sample sizes are warranted to identify the impact of tumor budding on the occurrence of distant metastasis.

In conclusion, histologic variants are not uncommonly detected in distant metastatic urothelial carcinomas. Despite its rarity, the histologic variants present in primary tumors are mostly maintained in metastatic tumors. High-grade tumor budding, which is frequently found in primary tumors, seems to play a significant role in the development of distant metastasis. Therefore, it may be useful to evaluate the presence of histologic variants and tumor budding in urothelial carcinomas of the urinary bladder to predict distant metastasis, even in non-muscle invasive urothelial carcinomas. 


\section{Ethics Statement}

All procedures performed in this study were approved by the Institutional Review Board at Ewha Womans University Mokdong Hospital (IRB No. 2019-07-013) in accordance with the Helsinki Declaration as revised in 2013. Formal written informed consent was waived by the IRB.

\section{ORCID}

Youngeun Yoo https://orcid.org/0000-0003-4855-3870 Junghye Lee https://orcid.org/0000-0001-8454-4360

Heae Surng Park https://orcid.org/0000-0003-1849-5120

Min-Sun Cho https://orcid.org/0000-0001-8772-9686

Sun Hee Sung https://orcid.org/0000-0001-9345-1131

Sanghui Park https://orcid.org/0000-0003-3837-8677

Euno Choi https://orcid.org/0000-0002-9284-9276

\section{Author Contributions}

Conceptualization: YY, JL, HSP, MSC, SHS, SP, EC. Data Curation: YY, JL. Formal analysis: YY, JL EC. Investigation: YY, EC. Methodology: SP, EC. Project administration: SP, EC. Resources: HSP, MSC, SHS. Supervision: SP, EC. Validation: EC. Visualization: YY, EC. Writing—original draft: EC. Writing - review \& editing: YY, JL, HSP, MSC, SHS, SP, EC. Approval of final manuscript: all authors.

\section{Conflicts of Interest}

The authors declare that they have no potential conflicts of interest.

\section{Funding Statement}

No funding to declare

\section{References}

1. Bray F, Ferlay J, Soerjomataram I, Siegel RL, Torre LA, Jemal A. Global cancer statistics 2018: GLOBOCAN estimates of incidence and mortality worldwide for 36 cancers in 185 countries. CA Cancer J Clin 2018; 68: 394-424.

2. Lopez-Beltran A, Henriques V, Montironi R, Cimadamore A, Raspollini MR, Cheng L. Variants and new entities of bladder cancer. Histopathology 2019; 74: 77-96.

3. Warrick JI. Clinical significance of histologic variants of bladder cancer. J Natl Compr Canc Netw 2017; 15: 1268-74.

4. Moch H, Humphrey PA, Ulbright TM, Reuter VE. WHO classification of tumours ot the uinary system and male genital organs. 4th ed. Lyon: International Agency for Research on Cancer, 2016; 77-98.

5. Moschini M, D'Andrea D, Korn S, et al. Characteristics and clinical significance of histological variants of bladder cancer. Nat Rev Urol 2017; 14: 651-68.

6. Amin MB. Histological variants of urothelial carcinoma: diagnostic, therapeutic and prognostic implications. Mod Pathol 2009; 22 Suppl 2: S96-118.

7. Liu Y, Bui MM, Xu B. Urothelial carcinoma with squamous differentiation is associated with high tumor stage and pelvic lymphnode metastasis. Cancer Control 2017; 24: 78-82.

8. Perepletchikov AM, Parwani AV. Micropapillary urothelial carcinoma: clinico-pathologic review. Pathol Res Pract 2009; 205: 807-10.

9. Keck B, Wach S, Stoehr R, et al. Plasmacytoid variant of bladder cancer defines patients with poor prognosis if treated with cystectomy and adjuvant cisplatin-based chemotherapy. BMC Cancer 2013; 13: 71 .
10. Sanfrancesco J, McKenney JK, Leivo MZ, Gupta S, Elson P, Hansel DE. Sarcomatoid urothelial carcinoma of the bladder: analysis of 28 cases with emphasis on clinicopathologic features and markers of epithelial-to-mesenchymal transition. Arch Pathol Lab Med 2016; 140: 543-51.

11. Venyo AK. Nested variant of urothelial carcinoma. Adv Urol 2014; 2014: 192720.

12. Lorenzo Soriano L, Ordaz Jurado G, Pontones Moreno JL, et al. Tumor budding: prognostic value in muscle-invasive bladder cancer. Urology 2019; 130: 93-8.

13. Fukumoto K, Kikuchi E, Mikami S, et al. Tumor budding, a novel prognostic indicator for predicting stage progression in T1 bladder cancers. Cancer Sci 2016; 107: 1338-44.

14. Ueno H, Murphy J, Jass JR, Mochizuki H, Talbot IC. Tumour 'budding' as an index to estimate the potential of aggressiveness in rectal cancer. Histopathology 2002; 40: 127-32.

15. Lugli A, Karamitopoulou E, Zlobec I. Tumour budding: a promising parameter in colorectal cancer. Br J Cancer 2012; 106: 1713-7.

16. Salhia B, Trippel M, Pfaltz K, et al. High tumor budding stratifies breast cancer with metastatic properties. Breast Cancer Res Treat 2015; 150: 363-71.

17. Kemi N, Eskuri M, Ikalainen J, Karttunen TJ, Kauppila JH. Tumor budding and prognosis in gastric adenocarcinoma. Am J Surg Pathol 2019; 43: 229-34.

18. Grigore AD, Jolly MK, Jia D, Farach-Carson MC, Levine H. Tumor budding: the name is EMT. Partial EMT. J Clin Med 2016; 5: 51.

19. Manig L, Kasmann L, Janssen S, Rades D. Predicting survival after irradiation of metastases from transitional carcinoma of the bladder. Anticancer Res 2016; 36: 6663-5.

20. Shelley MD, Cleves A, Wilt TJ, Mason MD. Gemcitabine chemotherapy for the treatment of metastatic bladder carcinoma. BJU Int 2011; 108: 168-79.

21. Sood S, Paner GP. Plasmacytoid urothelial carcinoma: an unusual variant that warrants aggressive management and critical distinction on transurethral resections. Arch Pathol Lab Med 2019; 143: 1562-7.

22. Ricardo-Gonzalez RR, Nguyen M, Gokden N, Sangoi AR, Presti JC Jr, McKenney JK. Plasmacytoid carcinoma of the bladder: a urothelial carcinoma variant with a predilection for intraperitoneal spread. J Urol 2012; 187: 852-5.

23. Bochner BH, Hansel DE, Efstathiou JA, et al. Urinary bladder. In: Amin MB, ed. AJCC cancer staging manual. 8th ed. New York: Springer, 2017; 346-55.

24. Lugli A, Kirsch R, Ajioka Y, et al. Recommendations for reporting tumor budding in colorectal cancer based on the International Tumor Budding Consensus Conference (ITBCC) 2016. Mod Pathol 2017; 30: 1299-311.

25. Shankar PR, Barkmeier D, Hadjiiski L, Cohan RH. A pictorial review of bladder cancer nodal metastases. Transl Androl Urol 2018; 7: 804-13.

26. MacVicar AD. Bladder cancer staging. BJU Int 2000; 86 Suppl 1: 111-22.

27. Jessen C, Agerbaek M, Von Der Maase H. Predictive factors for response and prognostic factors for long-term survival in consecutive, single institution patients with locally advanced and/or metastatic transitional cell carcinoma following cisplatin-based chemotherapy. Acta Oncol 2009; 48: 411-7.

28. Wallmeroth A, Wagner U, Moch H, Gasser TC, Sauter G, Mihatsch 
MJ. Patterns of metastasis in muscle-invasive bladder cancer (pT24): an autopsy study on 367 patients. Urol Int 1999; 62: 69-75.

29. Shinagare AB, Ramaiya NH, Jagannathan JP, Fennessy FM, Taplin ME, Van den Abbeele AD. Metastatic pattern of bladder cancer: correlation with the characteristics of the primary tumor. AJR Am J Roentgenol 2011; 196: 117-22.

30. Melms JC, Thummalapalli R, Shaw K, et al. Alpha-fetoprotein (AFP) as tumor marker in a patient with urothelial cancer with exceptional response to anti-PD-1 therapy and an escape lesion mimic. J Immunother Cancer 2018; 6: 89.

31. El-Bahrawy M. Alpha-fetoprotein-producing non-germ cell tumors of the urological system. Rev Urol 2011; 13: 14-9.

32. Klaile Y, Schlack K, Boegemann M, Steinestel J, Schrader AJ, Krab- be LM. Variant histology in bladder cancer: how it should change the management in non-muscle invasive and muscle invasive disease? Transl Androl Urol 2016; 5: 692-701.

33. Nigwekar P, Amin MB. The many faces of urothelial carcinoma: an update with an emphasis on recently described variants. Adv Anat Pathol 2008; 15: 218-33.

34. Jayasinghe C, Simiantonaki N, Kirkpatrick CJ. Histopathological features predict metastatic potential in locally advanced colon carcinomas. BMC Cancer 2015; 15: 14

35. Matthews PN, Madden M, Bidgood KA, Fisher C. The clinicopathological features of metastatic superficial papillary bladder cancer. J Urol 1984; 132: 904-6. 